\title{
COVID-19 and Crimean-Congo Hemorrhagic Fever: Is there any Similarity in Chest Radiology?
}

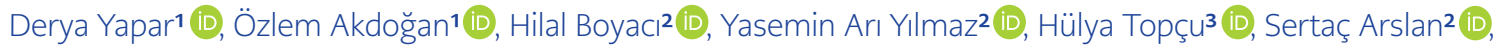 \\ Burak Yılmaz² (D), Gamze Kodalak4 (D), Kazım Çebi5 (D), Hüseyin Kayadibi6,7 (D), Aysel Kocagül Çelikbaş (iD, \\ Meral Gülhan² (iD), Nurcan Baykam¹ (iD) \\ 1 Department of Infectious Diseases and Clinical Microbiology, Hitit University School of Medicine, Çorum, Turkey \\ 2 Department of Chest Diseases, Hitit University School of Medicine, Çorum, Turkey \\ 3 Department of Anesthesiology, Hitit University School of Medicine, Çorum, Turkey \\ 4 Department of Internal Medicine, Hitit University School of Medicine, Çorum, Turkey \\ ${ }^{5}$ Department of Infectious Diseases and Clinical Microbiology, Hitit University Çorum Erol Olçok Education and Training Hospital, Çorum, Turkey \\ 6 Department of Medical Biochemistry, Hitit University School of Medicine, Çorum, Turkey \\ 7 Department of Medical Biochemistry, Eskişehir Osmangazi University, School of Medicine, Eskişehir, Turkey
}

\begin{abstract}
Objective: While the coronavirus disease 2019 (COVID-19) pandemic was continuing at full speed, patients with Crimean-Congo hemorrhagic fever (CCHF), which is endemic in our region, apply to the emergency department simultaneously. The presence of computed tomography (CT) lesions suggesting COVID-19 in some CCHF patients has brought to our mind the question of whether there is CCHF lung involvement even though respiratory symptoms are not at the forefront.

Methods: In this study, the findings of chest CT, demographic data and clinical symptoms of cases who had thorax tomography scan with suspicion of COVID-19 in the emergency department in the spring and summer of 2020 and were diagnosed with CCHF as a result of the evaluation and followed up in our clinic were compared with the findings of COVID-19 cases that were hospitalized and treated in the same period.

Results: Seventy-seven COVID-19 and 25 CCHF cases were included in the study. Myalgia, headache, diarrhea, nausea and vomiting were significantly higher in CCHF patients ( $p$ $<0.05)$. Cough was significantly more common in COVID-19 patients $(p=0.034)$. Groundglass opacity (GGO) was the most common tomography finding in CCHF, and cases without lung involvement were significantly higher $(p=0.001)$. GGO, consolidation, vascularization, atelectasis band, reverse halo, air-bubble, nodule were significantly high in COVID-19 patients.

Conclusion: During the epidemic period, no pathological finding was found in thoracic CT in most of the CCHF cases, and the presence of involvement in the lung tomography in cases with similar clinical and laboratory findings should primarily suggest the diagnosis of COVID-19.
\end{abstract}

Keywords: coronavirus disease 2019, COVID-19, Crimean-Congo hemorrhagic fever (CCHF), computed tomography (CT), ground-glass opacity (GGO)
Corresponding Author:

Derya Yapar

E-mail:

drderyayapar@hotmail.com

Received: August 21, 2021

Accepted: December 04, 2021

Published: February 14, 2022

\section{Suggested citation:}

Yapar D, Akdoğan Ö, Boyacı H, Arı-Yılmaz Y, Topçu H,

Arslan S, et al. COVID-19 and Crimean-Congo hemorrhagic fever: Is there any similarity in chest radiology? Infect Dis Clin Microbiol. 2022; 1.

DOI: 10.36519/idcm.2022.81 


\section{INTRODUCTION}

$\perp$ The number of cases of coronavirus disease 2019 (COVID-19) continues to threaten the world by increasing day by day. COVID-19 primarily affects the respiratory system and the most common clinical symptoms are fever, cough, dyspnea, headache, myalgia, and malaise (1). In the diagnosis of COVID-19, real-time reverse transcription-polymerase chain reaction (RT-PCR) of viral nucleic acid is considered the reference standard.

Duration of nasopharyngeal RT-PCR positivity is variable, but the probability of nasopharyngeal SARS-COV-2 RT-PCR positivity of COVID-19 patients decreases by time, especially at the second week of the disease and false-negative results could be seen. To avoid those false-negative results of PCR testing in patients with pneumonia, lower respiratory tract specimens can be an option for PCR testing, but obtaining a lower respiratory tract specimen is generally impossible due to the unproductive cough pattern of patients with COVID-19. Additionally, false-negative PCR-test results could also be seen due to technically incorrect specimen collection. All of them could lead to delays in the diagnosis of COVID-19. Thorax CT, which is a highly sensitive method (98\%), is used in the diagnosis of cases in which PCR was found to be negative (3). It has been reported that even asymptomatic cases may have lesions in thoracic CT (4).

Ten days after the first COVID-19 case reported in our country, COVID-19 cases started to be seen in Çorum. When the histories and examinations of some patients admitted to the COVID-19 services of our hospital were examined, it was seen that they were compatible with CCHF. The diagnosis of the cases was confirmed by CCHF PCR or CCHF Ig M positivity. In our study, the clinical findings of CCHF cases hospitalized with the pre-diagnosis of COVID-19 and the thorax CT results obtained during their hospitalization were compared with the data of COVID-19 cases.

\section{MATERIALS AND METHODS \\ Study Design}

In this retrospective cross-sectional study, between March 2020 and July 2020, COVID-19 and CCHF cases, tomographies were performed for differential diagnosis in the emergency service of Hitit University Çorum Erol Olçok Training and Research Hospital, then hospitalized and followed up and treated in the infectious diseases and clinical microbiology clinic, older than 18 years, diagnosis proven by PCR positivity were included. Demographic data, clinical findings and thorax CT involvement of the cases were compared. For the study, the necessary approvals were obtained from the ethics committee with May 05, 2020 date and number 233 and from the Ministry of Health with the date May 04, 2020, and number 2020-05-04T01-29-46.

\section{Data Collection}

The diagnosis of CCHF cases was made by PCR and/ or CCHF Ig M positivity in the serum. Diagnostic tests were performed at the Republic of Turkey Ministry of Health, Ankara Public Health Virology Laboratory.

Combined oropharynx and deep nasal swab samples taken for the real-time PCR (RT-PCR) test used in the diagnosis of COVID-19 were stored in viral transport media until the time of study and at +4 ${ }^{\circ} \mathrm{C}$ when necessary. COVID-19 PCR test was studied in the molecular laboratory of Hitit University Faculty of Medicine, Department of Medical Microbiology. Bio-Speedy® SARS-CoV-2 (2019-nCoV) qPCR detection kit (Bioeksen Inc., Turkey) for manual extraction, LightCycler® 96 RT-PCR system (Roche


PCR system (Anatolia Geneworks) and Bio-Speedy® COVID-19 RT-qPCR detection kit version 3-1000 Rxn (Bioeksen Inc., Turkey) were used for RT-PCR following the manufacturer's instructions.

Clinical, laboratory and CT findings of the cases were evaluated retrospectively. CTs were taken at the end of inspirium while the patients were in the supine position. Thorax CTs were evaluated by a

HIGHLIGHTS

- COVID-19 and CCHF are viral diseases with similar pathogenesis but different organ involvement.

- Lung involvement is rare in CCHF, so it does not appear to be necessary for the diagnosis of CCHF. 
clinically experienced pulmonologist. Lung lesions were defined as ground-glass opacity (GGO), consolidation, increased vascularization in consolidation, mosaic attenuation, atelectasis-atelectatic band, septal thickening, mediastinal lymphadenopathy (LAP), reverse halo, air-bubble, parallel band, emphysema, nodule.

\section{Statistical Analysis of Data}

SPSS Statistics for Windows, Version 21.0 (Armonk,

Table 1. Comparison of symptoms between COVID-19 and CCHF.

\begin{tabular}{|l|c|c|c|}
\hline & $\begin{array}{c}\text { COVID-19 } \\
\mathrm{n}(\%)\end{array}$ & $\begin{array}{c}\text { CCHF } \\
\mathrm{n}(\%)\end{array}$ & $p$ \\
\hline Fever & $46(59.7)$ & $21(75)$ & 0.150 \\
\hline Cough & $24(31.2)$ & $3(10.7)$ & 0.034 \\
\hline Myalgia & $19(24.7)$ & $25(89.3)$ & $<0.001$ \\
\hline Headache & $10(13)$ & $12(42.9)$ & 0.001 \\
\hline $\begin{array}{l}\text { Diarrhea-nausea- } \\
\text { vomiting }\end{array}$ & $7(9.1)$ & $9(32.1)$ & 0.011 \\
\hline Sore throat & $9(11.7)$ & $1(3.6)$ & 0.283 \\
\hline Chest pain & $1(1.3)$ & $3(10.7)$ & 0.057 \\
\hline
\end{tabular}

CCHF: Crimean-Congo hemorrhagic fever
NY: IBM Corp.) was used for statistical analysis of the data obtained in the study. Data of continuous variables were expressed as mean value, standard deviation and data of categorical variables were expressed as percentages. In the analyzes comparing the two groups, we used the Mann-Whitney U test for continuous variables, the Chi-square test and Fisher's exact test for categorical variables, and Spearman's correlation test for correlations. The results were evaluated at a 95\% confidence interval and the statistical significance was set as $p<0.05$.

\section{RESULTS}

During the study period, 28 CCHF cases were followed up in our clinic. A total of three CCHF cases were excluded from the study: a patient who was hospitalized with the diagnosis of CCHF and died with the picture of "disseminated intravascular coagulation (DIC) and multiple organ dysfunction syndrome (MODS)" within the first few hours of hospitalization, a patient who did not have thorax CT because he was under the age of 20 and a patient who was hospitalized with a diagnosis of CCHF and did not require a CT scan because there was no suspicion of COVID. As a result, 77 COVID-19 and 25 CCHF cases were included in the study.

Table 2. Comparison of laboratory parameters between COVID-19 and CCHF.

\begin{tabular}{|c|c|c|c|c|c|c|c|}
\hline & \multicolumn{3}{|c|}{ COVID-19 } & \multicolumn{3}{|c|}{$\mathrm{CCHF}$} & \multirow{2}{*}{$p$} \\
\hline & Mean & SD & Median & Mean & SD & Median & \\
\hline WBC $\left(10^{3} / \mu \mathrm{L}\right)$ & 6087.5 & 2102.02 & 6220 & 3794.29 & 2447.83 & 3340 & $<0.001$ \\
\hline Neutrophil $\left(10^{3} / \mu \mathrm{L}\right)$ & 3881.84 & 1849.11 & 3545 & 2878.57 & 2116.09 & 2390 & 0.002 \\
\hline Lymphocyte $\left(10^{3} / \mu \mathrm{L}\right)$ & 1633.16 & 797.12 & 1420 & 646.07 & 493.94 & 460 & $<0.001$ \\
\hline Platelet $\left(10^{3} / \mu \mathrm{L}\right)$ & $213,674.3$ & 76,822 & 209,000 & $110,285.7$ & $62,249.71$ & 124,500 & $<0.001$ \\
\hline Fibrinogen (mg/dL) & 403.32 & 134.14 & 380 & 267.20 & 59.99 & 272 & $<0.001$ \\
\hline INR & 1.14 & 0.20 & 1.1 & 1.17 & 0.19 & 1.135 & 0.221 \\
\hline AST (U/L) & 29.58 & 23.55 & 23.5 & 224.14 & 496.02 & 62 & $<0.001$ \\
\hline ALT (U/L) & 25.71 & 25.23 & 17 & 87.11 & 186.34 & 43 & 0.0001 \\
\hline $\mathrm{LDH}(\mathrm{U} / \mathrm{L})$ & 238.13 & 75.97 & 231 & 597 & 772.42 & 305.5 & 0.0001 \\
\hline CK (U/L) & 148.93 & 194.76 & 78.5 & 662.07 & 1029.17 & 287.5 & $<0.001$ \\
\hline
\end{tabular}

ALT: Alanine aminotransferase, AST: Aspartate aminotransferase, CK: Creatine kinase,

INR: International normalized ratio, LDH: Lactate dehydrogenase, SD: Standard deviation, WBC: White blood cells, CCHF: Crimean-Congo hemorrhagic fever. 
No statistically significant difference was found between CCHF and COVID-19 cases in terms of gender $(p>0.05)$. The median age was 56.5 years in CCHF cases and 54 years in COVID-19 cases. There was no difference between the two groups in terms of the presence of underlying pulmonary disease (asthma / chronic obstructive pulmonary disease [COPD]) ( $p$ $>0.05)$.

Myalgia, headache, diarrhea, nausea and vomiting were significantly higher in CCHF patients $(p<$ 0.05). In COVID-19 patients, the incidence of cough was significantly higher as expected $(p=0.034)$. Hemoptysis was not observed in any groups. The clinical findings of the patients are presented in Table 1.

Laboratory findings such as leukopenia, thrombocytopenia, low fibrinogen, AST, ALT, LDH, CPK increase, typical in CCHF cases, made a significant difference to distinguish the cases from COVID-19 cases (Table 2).

Pulmonary involvement was not detected in the PA chest X-ray of 21 (84\%) CCHF cases included in the study. The difference was significant when compared with the COVID-19 cases $(28.6 \%)(p=0.001)$.

Ground-glass opacity (GGO), defined as a specific CT finding in the diagnosis of COVID 19 cases, was detected in $28 \%$ of CCHF patients. This finding was significantly higher in COVID-19 cases $(p=0.0003)$ (Table 3).

Two of the CCHF patients (8\%) had pneumonia corresponding to their clinical findings. Consolidation was detected in the tomography of these cases. Also, consolidation was detected in 14 (18.2\%) COVID-19 patients. There was no statistically significant difference between the two groups ( $p>0.745)$.

The tomography findings encountered in COVID-19 cases, such as reverse halo, air-bubble, nodule, were not detected in CCHF cases. The difference was significant between the two groups. Also, atelectasis, emphysema, and bronchial dilatation were not detected in CCHF patients. Other tomography findings reported in COVID-19 patients and the comparison with CCHF are shown in Table 3.

\section{DISCUSSION}

CCHF is a tick-borne zoonotic disease caused by an RNA virus within the nairovirus group of the Bunyaviridae family. It was first described in the Soviet Union after an epidemic in Crimea in 194445 , and after it was understood that the factor was identical with the virus isolated from a febrile patient in Congo in 1956, the disease was named as "Crimean-Congo hemorrhagic fever". Currently, CCHF cases are reported in many countries, including Turkey, in Asia, Southern and Eastern Europe, Africa and the Middle East. The disease is seen as endemic in our country. The first CCHF cases were noticed in the provinces of Tokat, Sivas, Yozgat, Artvin, Gümüşhane, Giresun and Trabzon in 2002. The province of Çorum, where our university is located, is also located in the Kelkit Valley, which is a hyperendemic region in terms of CCHF. The disease is seen in the spring and summer months when ticks are active, and CCHF cases are followed up in our hospital every year during these months. It may present with different clinical pictures ranging from asymptomatic cases to hypotension, multiple

Table 3. Comparison of tomography findings between groups.

\begin{tabular}{|c|c|c|c|}
\hline Lung lesions & $\begin{array}{c}\text { COVID-19 } \\
\text { n (\%) }\end{array}$ & $\begin{array}{l}\text { CCHF } \\
\mathrm{n}(\%)\end{array}$ & $P$ \\
\hline \multicolumn{4}{|l|}{ PA Chest X-ray } \\
\hline No radiological findings & $22(28.6)$ & $21(84)$ & $<0.001$ \\
\hline \multicolumn{4}{|c|}{ Chest tomography findings } \\
\hline GGO & $55(71.4)$ & $7(28)$ & $<0.001$ \\
\hline Consolidation & $14(18.2)$ & $2(8)$ & 0.745 \\
\hline Septal thickening & $6(7.8)$ & $1(4)$ & $>0.999$ \\
\hline Reverse halo & $14(18.2)$ & $0(0)$ & 0.019 \\
\hline Air-bubble & $24(31.2)$ & $0(0)$ & 0.001 \\
\hline Nodule & $15(19.5)$ & $0(0)$ & 0.019 \\
\hline Mediastinal LAP & $3(3.9)$ & $1(4)$ & $>0.999$ \\
\hline Emphysema & $6(7.8)$ & $0(0)$ & 0.332 \\
\hline Bronchial dilatation & $9(11.7)$ & $0(0)$ & 0.108 \\
\hline Nodule & $55(70.4)$ & $0(0)$ & $<0.001$ \\
\hline
\end{tabular}

GGO: Ground-glass opacity, LAP: Lymphadenopathy, PA: Posteroanterior, CCHF: Crimean-Congo hemorrhagic fever. 
organ failure, shock, DIC, bleeding and death. The mortality is reported as between $5-30 \%$; however, the mortality in our country is around 5\% (5).

While the COVID-19 pandemic were at full tilt, CCHF cases, too, applied to the emergency service of our hospital in the spring and summer of 2020. Healthcare professionals working in the emergency department made an intense effort not to bypass the COVID-19 cases because this process coincided with the peak period of COVID-19 cases. During the differentiation of CCHF cases from COVID-19, it was observed that thorax CT was performed in many cases with the pre-diagnosis of COVID-19. The cases can be confused with COVID-19 because the symptoms are nonspecific in the early period of CCHF. However, it is possible to distinguish CCHF cases from COVID-19 in the prehemorrhagic and hemorrhagic periods, when clinical and laboratory findings are established, even without tomography. As a matter of fact, in the presented cases, frequent findings in CCHF cases such as myalgia, headache, diarrhea, nausea and vomiting were found to be significantly higher compared to COVID-19 cases. Hemoptysis is generally not one of the symptoms seen in the course of mild CCHF cases and COVID-19 (6). It was not detected in our cases, either.

CCHF-specific leukopenia, thrombocytopenia can also be seen in COVID-19 cases. However, blood leukocyte, lymphocyte and platelet counts were significantly lower among cases with CCHF.

We also found that serum aminotransferase levels of patients with CCHF were significantly higher than patients with COVID-19 at the emergency unit admission. It is known that patients with or without pre-existing liver disease may present with elevated aminotransferases in the setting of COVID-19 and agents that were used such as favipiravir and colchicine could also cause AST and ALT increase. But in COVID-19, serum aminotransferase levels, a predictor of severe disease, increase mainly at the second week of the disease. Consequently, our results suggest that these parameters can be used to distinguish two diseases during emergency admissions. Since the primary target of COVID-19 is the lungs, high LDH is seen in the majority of cases. Among CCHF cases, elevated LDH is one of the parameters evaluated during the diagnosis and follow-up phase. LDH elevation was significantly higher in our cases, too. High CPK is a finding that also supports the diagnosis of CCHF. High CPK is rarely seen in COVID-19 cases $(7,8,9)$.

Most of the patients diagnosed with CCHF applied to the hospital with the complaint of fever and had a thorax CT scan in the emergency department to rule out COVID-19 as it was the early period of the pandemic. Four of these cases were hospitalized in the COVID-19 services and one in the COVID-19 intensive care unit, and the next day, during the visit of the infectious disease specialist, they were transferred to the clean area with the pre-diagnosis of CCHF.

CCHF primarily involves the vascular endothelium, bone marrow, and reticuloendothelial system. Pulmonary involvement is not a routine finding in CCHF cases. In the later stages of the disease, clinical and radiological pulmonary findings can be seen in cases, such as intra-alveolar bleeding, transfusion-related acute lung injury (TRALI) that develops during intensive blood product replacement, and hospital-acquired pneumonia. There are few studies about pulmonary involvement in CCHF in the literature. Intra-alveolar bleeding, hemothorax and acute respiratory distress syndrome (ARDS) were reported. Symptoms such as dyspnea, chest pain, hemoptysis are seen especially in patients in the hemorrhagic phase, and these cases are often mortal $(8,10,11)$.

A study in which thorax CT findings of CCHF patients were examined reported that GGO was observed in $57.5 \%$ of the cases. The majority of the patients with GGO were in the hemorrhagic period, and this finding was bilateral in $42.5 \%$ and unilateral in $15 \%$. Consolidation was detected in $25 \%$ of the cases in the same series, and $20 \%$ of them were bilateral (12). GGO was not detected in $72 \%$ of our CCHF cases. This may be related to the patients' admission to the hospital in the prehemorrhagic period. Consolidation rate was $8 \%$. GGO was the most common finding in COVID-19 cases. GGO lesions gradually turn into crazy paving pattern and then consolidation. Because consolidations appeared in the late period of COVID-19 pneumonia, 
no consolidation was observed in early tomographies at a level that would make a difference from CCHF (2).

\section{CONCLUSIONS}

In conclusion it seems possible to distinguish CCHF cases from COVID-19 cases with clinical and laboratory findings. Our results suggest that, in both COVID-19 and CCHF endemic settings, presence of myalgia, headache, diarrhea, nausea and vomiting in a patient should recall CCHF to emergency unit physicians, and higher AST, ALT, LDH, CPK and lower blood leucocyte, lymphocyte and platelet lev- els should support this diagnosis. Our results also show once again seen that thorax CT, which is almost indispensable in the diagnosis of COVID-19, does not have a diagnostic place in CCHF.

The fact that the target organ in CCHF is the vascular bed, bone marrow and reticuloendothelial system increases the possibility of detecting lesions in thoracic CT, especially in the hemorrhagic phase, but this will not contribute to the patient's treatment other than detecting hemorrhage. In CCHF cases with concomitant pneumonia, it can be decided by PA chest radiography, so there is no need for lung tomography under emergency conditions.
Ethical Approval: The Ethics Committee of Hitit University approved the study on May 05, 2020 date and with the decision number of 233 and the Ministry of Health approved the study on May 04, 2020 and with the decision number of 2020-05-04T01-29-46.

Informed Consent: N/A

Peer-review: Externally peer-reviewed

Author Contributions: Concept - D.Y., N.B., M.G., A.K.Ç., Ö.A., S.A., H.B., Y.A.Y., G.K.; Design - D.Y., N.B., M.G., A.K.Ç., Ö.A., S.A., H.B., Y.A.Y.; Supervision - D.Y., Y.A.Y., H.B., B.K., G.K.; Fundings - D.Y., B.Y., G.K., H.B., S.A., Y.A.Y., H.K., K.Ç., Ö.A.; Materials - D.Y., B.Y., G.K.,
H.B., S.A., Y.A.Y., H.K., K.Ç., Ö.A.; Data Collection and/or Processing - D.Y., B.Y., G.K., H.B., S.A., Y.A.Y., H.K., K.Ç., Ö.A.; Analysis and/or Interpretation - D.Y., M.G., A.K.Ç., N.B., H.K., H.T., Ö.A.; Literature Review - D.Y., Ö.A., A.K.Ç., N.B., M.G., H.T., H.K., K.Ç.; Writer - D.Y., Ö.A., A.K.Ç., N.B., M.G., H.T., N.B., Y.A.Y., G.K., D.Y., S.A., M.G.; Critical Reviews - D.Y., Ö.A., N.B., Y.A.Y., H.T., B.Y., G.K., H.K., K.Ç., M.Ç., A.K.Ç., N.B.

Conflict of Interest: The authors declare no conflict of interest.

Financial Disclosure: The authors declared that this study has received no financial support.

\section{REFERENCES}

1 Pascarella G, Strumia A, Piliego C, Bruno F, Del Buono R, Costa F, et al. FE. COVID-19 diagnosis and management: a comprehensive review. J Intern Med. 2020;288(2):192-206. [CrossRef]

2 Pan F, Ye T, Sun P, Gui S, Liang B, Li L, et al. Time course of lung changes at chest CT during recovery from coronavirus disease 2019 (COVID-19). Radiology. 2020;295(3):715-21. [CrossRef]

3 Fang $\mathrm{Y}$, Zhang H, Xie J, Lin M, Ying L, Pang P, et al. Sensitivity of chest CT for COVID-19: comparison to RT-PCR. Radiology. 2020;296(2):E115-7. [CrossRef]

4 Pozzessere C, Rotzinger DC, Ghaye B, Lamoth F, Beigelman-Aubry C. Incidentally discovered COVID-19 pneumonia: the role of diagnostic imaging. Eur Radiol. 2020;30(9):5211-3. [CrossRef]

5 Nasirian $\mathrm{H}$. New aspects about Crimean-Congo hemorrhagic fever (CCHF) cases and associated fatality trends: A global systematic review and meta-analysis. Comp Immunol Microbiol Infect Dis. 2020;69:101429. [CrossRef]

6 Shi F, Yu Q, Huang W, Tan C. 2019 novel coronavirus (COVID-19) pneumonia with hemoptysis as the initial symptom: CT and clinical features. Korean J Radiol. 2020;21(5):537-40. [CrossRef]
7 Wan S, Xiang Y, Fang W, Zheng Y, Li B, Hu Y, et al. Clinical features and treatment of COVID-19 patients in northeast Chongqing. J Med Virol. 2020;92(7):797-806. [CrossRef]

8 Fillâtre P, Revest M, Tattevin P. Crimean-Congo hemorrhagic fever: An update. Med Mal Infect. 2019;49(8):574-85. [CrossRef]

9 Kayadibi H, Yapar D, Akdogan O, Ulusu NN, Baykam N. Hitit Index to distinguish patients with and without Crimean-Congo hemorrhagic fever. Ticks Tick Borne Dis. 2019;10(5):1035-40. [CrossRef]

10 Swanepoel R, Shepherd AJ, Leman PA, Shepherd SP, McGillivray GM, Erasmus MJ, et al. Epidemiologic and clinical features of Crimean-Congo hemorrhagic fever in southern Africa. Am J Trop Med Hyg. 1987;36(1):120-32. [CrossRef]

11 Sannikova IV, Pacechnikov VD, Maleev VV. [Respiratory lesions in Congo-Crimean hemorrhagic fever]. Ter Arkh. 2007;79(11):20-3. Russian.

12 Aktaş T, Aktaş F, Özmen Z, Altunkaş A, Kaya T, Demir O. Thorax CT findings in patients with Crimean-Congo hemorrhagic fever (CCHF). Springerplus. 2016;5(1):1823. [CrossRef] 